\title{
Caracterización silvícola de ñirantales del norte de la Patagonia para la gestión forestal sostenible
}

\author{
Silvicultural characterization of the mixed ñire forests of northern Patagonia \\ as a basis for sustainable forestry
}

\author{
José A Reque**, Mauro Sarasola ${ }^{\mathrm{b}}$ Javier Gyengec $^{\mathrm{c}}$, María Elena Fernández \\ *Autor de correspondencia: aUniversidad de Valladolid, Escuela Técnica Superior de Ingenierías Agrarias, \\ Avda. de Madrid 57, 34071 Palencia, España, Tel.: 34-979108422, fax: 34-979198401, requekch@pvs.uva.es \\ bInstituto Nacional de Tecnología Agropecuaria, INTA-EEA Bariloche, Argentina. \\ ${ }^{c}$ Consejo Nacional de Investigaciones Científicas y Técnicas (CONICET), INTA-EEA Bariloche, Argentina.
}

\begin{abstract}
SUMMARY
In spite of their wide geographical distribution and use, there are few publications about the silviculture of the forests with Nothofagus antarctica, locally named "ñirantales". The available publications report studies carried out in central to southern Patagonia, where "ñires" form forests of relatively tall trees. The traditional management of the ñire forests has been based in fuelwood production and silvopastoralism. Characterization of the forests of Lomatio-Nothofagetum antarcticae of the central basin of the Foyel River (Río Negro, Patagonia, Argentina) was carried out in order to provide methodological skills useful for the sustainable management of "ñirantales". We developed a typologic key, the silvicultural stand characterization of the different forest types present in the area and we established the local dendrometric equations for N. antarctica, Diostea juncea, Lomatia hirsuta, Schinus patagonicus. The studied forests showed a high variability between stands in close distance. Stem diameter distribution of the different forest types indicate that they are even aged groves in an intermediate development phase. Based on the current development stage of the stands, we suggest that it is possible to apply forestry models based on density regulation and complete canopy cover. Based on our results, we preliminary suggest that the implementation of silvicultural systems based on wood extraction can be seen as an alternative to current silvopastoral systems.
\end{abstract}

Key words: Nothofagus antarctica, silviculture, forest structure, typology, Northern Patagonia.

\section{RESUMEN}

A pesar de la amplia distribución geográfica y uso de los ñirantales, son muy escasos los trabajos descriptivos sobre la silvicultura en estas comunidades forestales, centrándose principalmente en las zonas donde el ñire (Nothofagus antarctica) alcanza un mayor desarrollo arbóreo (centro y sur de la Patagonia). Tradicionalmente, el manejo de los ñirantales se ha basado en la obtención de leña y en la silvopascicultura. Con el objetivo de sentar bases metodológicas para la gestión forestal sostenible de los ñirantales se caracterizaron los bosques de Lomatio-Nothofagetum antarcticae de la cuenca central del río Foyel (Río Negro, Patagonia, Argentina), estableciendo una clave tipológica, así como la caracterización silvícola de los diferentes tipos de masa presentes en la zona y las ecuaciones dendrométricas locales para N. antarctica, Diostea juncea, Lomatia hirsuta y Schinus patagonicus. Los resultados indican que en la zona de estudio existe una marcada variabilidad y heterogeneidad espacial, apareciendo diferencias dasométricas entre rodales en muy corto espacio. Las distribuciones diamétricas de los distintos tipos de ñirantal muestran mayoritariamente formas principales de masa regulares en estado intermedio de desarrollo, abriéndose la posibilidad a considerar la implementación de modelos silvícolas basados en el manejo de la espesura y la ocupación completa del potencial productivo del sitio. Las variables dasométricas indican que -además del actual manejo silvopastoril- la implementación de modelos de gestión sostenible fundamentados en la obtención de biomasa leñosa puede ser una alternativa de manejo.

Palabras clave: Nothofagus antarctica, silvicultura, estructura forestal, tipología, Patagonia Norte. 


\section{INTRODUCCIÓN}

Uno de los ecosistemas forestales más característicos de los bosques caducifolios de la Patagonia andina de Argentina es aquel constituido por los bosques, chaparrales y montes bajos de ñire (Nothofagus antarctica (G. Forst.) Oerst.) (Veblen et al. 1996). Estos ecosistemas forestales se encuadran mayoritariamente en la provincia fitogeográfica subantártica (distrito del bosque semicaducifolio) definido por Cabrera (1976) y se desarrollan en una franja de accidentada topografía a ambos lados de la Cordillera de Los Andes (latitudes $37^{\circ} \mathrm{S}$ a $55^{\circ} \mathrm{S}$ ). El gradiente altitudinal en el que se desarrolla $N$. antarctica es muy amplio. Así, mientras en el norte de su área de distribución la especie se presenta a grandes altitudes donde llega a aparecer a $2.000 \mathrm{~m}$ s.n.m., los ñirantales forman el límite oceánico del bosque en Tierra de Fuego. Desde un punto de vista botánico los ñirantales se adscriben mayoritariamente a la clase Nothofagetea pumilionis antarcticae (Oberdorfer 1960).

Las actuales configuración y estructura de los bosques y montes bajos de ñire son mayoritariamente consecuencia de usos antrópicos implementados a partir de finales del siglo XIX con el asentamiento de población de origen europeo. Destacan el pastoreo, el uso del fuego con fines pascícolas y la tala de árboles, pudiéndose afirmar que los ñirantales son las comunidades forestales más intensamente explotadas en muchas zonas de la región patagónica (Montaña 1982). En este sentido, son numerosos los autores que consideran a los ñirantales como estados de regresión de bosques de ciprés de la cordillera (Austrocedrus chilensis (D. Don) Pic. Ser. et Bizz.) o coihue (Nothofagus dombeyi (Mirb.) Oerst.), actuando el ganado y el fuego como retardantes en los procesos sucesionales (Eskuche 1969, Veblen et al. 2003).

Aun presentando los bosques y chaparrales de ñire un área de distribución muy amplia, coincidente con la clase Nothofagetea pumilionis antarcticae (Eskuche 1969), los trabajos sobre la silvicultura del ñire son muy escasos, centrándose en las zonas en las que el ñire alcanza los mayores desarrollos arbóreos como en la provincia de Tierra de Fuego o en estudios silvopastoriles (Lencinas et al. 2002).

El principal aprovechamiento económico de los ñirantales es el silvopastoril. El pastoreo se realiza mayoritariamente en régimen extensivo de forma libre y continua, llegando a utilizarse los bosques de $N$. antarctica en zonas con marcados gradientes hídricos o térmicos como cuarteles de invernada o veranada (Somlo et al. 1997, Manacorda y Bonvissuto 2001, Hansen et al. 2005). En este sentido es importante la bibliografía técnica y científica en la que se estudia la relación entre la cobertura de copas arbórea y la producción forrajera, siendo un importante reto científico la definición de los grados de cobertura arbórea óptimos para las diferentes estacio- nes ecológicas de la Patagonia (Somlo y Bonvissuto 1995, SAyD 2005, Peri 2005).

El ñire presenta capacidad de reproducción vegetativa, habiéndose basado tradicionalmente la silvicultura de la especie en la regeneración asexual. Es por ello que otra de las bases a definir en la gestión sostenible de estos ecosistemas es la compatibilidad del uso silvopastoril con la regeneración natural. Nothofagus antarctica presenta comúnmente desarrollos en altura inferiores a $15 \mathrm{~m}$, por lo que los aprovechamientos forestales se suelen centrar en la obtención de leña y de piezas para postes. Las intensidades de corta aplicadas son muy variables, desde el apeo selectivo de pies muertos, hasta la corta a matarrasa. No obstante, la gradual asunción de la positiva correlación entre diferentes niveles de cobertura arbórea y la producción forrajera hace que las cortas de fuerte intensidad sean cada vez menos comunes (Peri $e t$ al. 2005).

Como fase previa al establecimiento de las bases para la gestión sustentable de los ñirantales se comienzan a realizar en la década de los ochenta del siglo XX trabajos tendientes a caracterizar su forma fisionómico-estructural. Anteriormente, los trabajos se habían centrado en el ámbito de la fitosociología, destacando las clásicas obras de Oberdorfer (1960) y de Eskuche (1968, 1969). Más recientemente deben ser citados los trabajos de Gajardo (1994), Veblen et al. (1996), Amigo y Ramírez (1998) y Eskuche (1999).

Analizando los patrones de distribución de las distintas especies y asociaciones vegetales en diferentes gradientes ambientales en bosques del centro-sur de Chile, y en un intento de incluir variables básicas para la silvicultura, Pauchard et al. (2006) incluyen en su estudio botánico mediciones propias de inventario forestal (diámetro normal, distribución diamétrica, etc.). Estos autores presentan las variables dasométricas básicas para los dos tipos fisonómico-estructurales en los que aparece ñire como especie dominante.

Entre los trabajos que describen y clasifican la variación fenotípica de los ñirantales destacan las publicaciones de Gómez y Gallopín (1982), de Montaña (1982) y de Ramírez et al. (1985). Dichos autores coinciden que $N$. antactica adopta diferentes formas biológicas, caracterizadas por el ambiente en el que vegetan. Así, Gómez y Gallopín (1982) describen para la cuenca superior del Río Manso (Provincia de Río Negro, Argentina) tres morfotipos: mesofanerofítico, microfanerofítico y nanofanerofítico. En el mismo sentido, Ramírez et al. (1985) clasifican tres tipos morfológicos para el centro-sur de Chile, atendiendo al desarrollo del sistema aéreo vegetativo: arborescente, arbustivo achaparrado y camefítico. Por último, el trabajo de Gajardo et al. (2005) establece una tipología de sitios forestales para la región de la Araucanía (Chile), ligando las diferentes asociaciones fitosociológicas descritas para la zona (entre las que aparecen ñirantales) con indicadores ecológicos del sitio. 
El carácter arbustivo o subarbóreo de una importante representación de los ñirantales (Oberdorfer 1960, Eskuche 1969, Veblen et al. 2003) hace que extensas áreas sean consideradas no inventariables cuantitativamente desde un punto de vista dasométrico. Así, en muchos de los trabajos en los que se establecen las bases para la silvicultura de estos ecosistemas queda excluida de inventariación forestal una muy notable representación de ñirantales, definiéndose, por ejemplo, diámetros mínimos inventariables de $5 \mathrm{~cm}$ para el sur de Chile (Pauchard et al. 2006) o de 7,5 cm para Tierra de Fuego (Fernández et al. 1995, Martínez-Pastur et al. 1995, Martínez-Pastur y Fernández 1995, Lencinas et al. 2002). Tampoco Collado (2001) llega a establecer una clasificación satelital completa de los ñirantales de Tierra de Fuego debido a su reducido desarrollo aéreo.

Uno de los ecosistemas forestales de ñire característico del noroeste de la Patagonia y frecuentemente excluido de inventariación forestal por su escaso desarrollo son los bosques y montes bajos de ñire clasificados como Lomatio-Nothofagetum antarcticae (alianza Nothofago Berberidion; orden Berberi-Nothofagetalia-antarcticae) (Eskuche 1969). Entre los principales intereses de estas comunidades está su carácter permanentemente antropogéneo, lo cual hace que se sitúen preferentemente en zonas pobladas o fácilmente accesibles para el hombre y su ganado. La situación física de estos montes, unida al escaso valor económico de su madera, ha llevado desde los años setenta del siglo XX a considerar la conveniencia de sustituir estos ecosistemas por repoblaciones forestales con coníferas exóticas de crecimiento rápido (Pseudotsuga menziesii ((Mirb.) Franco.), Pinus contorta (Dougl. ex Loud.), Pinus ponderosa (Dougl. ex Lawson), etc. En este sentido, Laclau (1997) estima que de las 50 mil hectáreas repobladas con coníferas alóctonas en la zona, entre un $30 \%$ y un $40 \%$ se efectuó sobre masas nativas de ñire o ciprés de la cordillera. El fuerte empuje que está viviendo la silvicultura de las masas artificiales de coníferas contrasta con el escaso nivel de conocimientos sobre la silvicultura del bosque caducifolio nativo en la zona. Hasta finales del siglo XX no empieza a estudiarse en el noroeste patagónico la silvicultura del bosque nativo de Nothofagaceae (Chauchard et al. 1995ab, Gallo et al. 2000, Ipinza et al. 2000). En ninguno de los trabajos se incluye a N. antarctica, centrándose las publicaciones en lenga (Nothofagus pumilio (Poepp et Endl.) Krasser), raulí (N. alpina Poepp. et Endl. = N. nervosa (Phil.) Krasser), roble pellín ( $N$. obliqua (Mirb.) Oerst.) y coihue (N. dombeyi).

Con el objetivo de sentar bases metodológicas para el desarrollo de la gestión forestal sostenible de los ñirantales, en este trabajo se clasifican silvícolamente los bosques y montes bajos de Lomatio-Nothofagteum antarcticae del norte de la Patagonia argentina. Como objetivos específicos se considera: (a) establecer una clave tipoló- gica de ñirantales arborescentes de fácil uso e interpretación, (b) caracterizar silvícolamente los diferentes tipos de masa estableciendo los valores dasométricos medios de cada tipo estructural y (c) valorar posibles escenarios silvícolas distintos a los tradicionales usos silvopascícolas o de aprovechamiento de leña en monte bajo.

\section{MÉTODOS}

Zona de estudio. La zona de estudio ocupa aproximadamente 100 mil hectáreas de ambientes dominados por ñire en la cuenca del río Foyel (provincia de Río Negro, Argentina), (4139'89' S, 71³3'28', 645 m s.n.m.). La influencia antrópica en la configuración del paisaje es muy evidente, donde el fuego ha sido un elemento recurrente desde principios del siglo XX (Willis 1914), modificando y determinado la dinámica de los bosques de la zona. Tal autor describe la zona como quemada de forma intencionada prácticamente en su totalidad. La implementación de usos y aprovechamientos ganaderos se remonta al mismo periodo, siendo la zona actualmente aprovechada mediante la aplicación de sistemas silvopastoriles (Somlo et al. 1997, Manacorda y Bonvissuto 2001). El clima de la zona se caracteriza por presentar rasgos propios de la provincia fitogeográfica Austral (distrito del Bosque Caducifolio): precipitación media anual es de 920 $\mathrm{mm}$, temperatura media anual de $9,7^{\circ} \mathrm{C}$ (rangos climáticos medios entre $16,7{ }^{\circ} \mathrm{C}$ y $3,1{ }^{\circ} \mathrm{C}$ ) y probabilidad de heladas durante todo el año.

Estructura forestal. Las comunidades dominadas por ñire se encuadran dentro de los bosques bajos de LomatioNothofagetum (Eskuche 1969) y ocupan, fundamentalmente, fondos de valles y laderas bajo 1.200 m s.n.m. Nothofagus antarctica, rara vez supera $15 \mathrm{~m}$ de altura, siendo más común alturas de 6 a 12 metros. Además de ñire, entre las especies arbóreas representativas de la comunidad forestal, se citan el radal (Lomatia hirsuta (Lam.) Diels ex Macbr.) y el maitén (Maytenus boaria Mol.). De especial interés en la zona es el desarrollo que puede alcanzar la verbenacea retamo (Diostea juncea (Gillies et Hook.) Miers), la cual puede llegar al estrato arbóreo. Dentro de las arbustivas destacan: laura (Schinus patagonicus (Phil.) Johnst.), zarzaparrilla (Ribes magallanicum Speg.), calafate (Berberis buxifolia Lam.) y michai (B. darwinii Hook.). Representando microambientes de mayor humedad aparece la caña coligüe (Chusquea culeou Desv.) y diferentes variantes del género Pernettya (Somlo et al. 1997).

Selección de rodales. La selección de rodales de estudio se basó en la estratificación y la cartografía de tipos de masa establecidas para la cuenca central del río Foyel por Rusch (2005) y Lantschner (2005). Como fase previa para posteriores censos ornitológicos se definen en estos 
trabajos cuatro estructuras forestales de ñirantales, considerando la cobertura arbórea y la composición específica fruto del accionar antropogénico en la región norte de la Patagonia: (a) bosque de ñire cerrado, (b) bosque de ñire semiabierto, (c) bosque mixto cerrado y (d) bosque mixto semiabierto. De acuerdo con esta tipología estructural, Lantschner (2005) replantea en un muestreo dirigido 41 rodales para realizar en ellos censos de aves. Tomando la base cartográfica de replanteo de parcelas de los censos ornitológicos de Lantschner (2005) se seleccionaron en el presente trabajo 17 rodales repartidos en forma aleatoria entre las cuatro estructuras de ñirantales definidas por Rusch (2005).

Siguiendo un rumbo que recorriera completamente el lado mayor de rodal se replanteó una parcela en el centro del mismo. Se establecieron parcelas de inventario de $250 \mathrm{~m}^{2}$ para bosques donde la densidad de árboles por hectárea fue mayor a 2.500 y parcelas de $500 \mathrm{~m}^{2}$ para menores densidades.

Funciones dendrométricas. Para el establecimiento de las relaciones dendrométricas se apearon 36 árboles de las cuatro especies principales (cuadro 1). En total se seleccionaron diez individuos de Nothofagus antarctica y Diostea juncea, y ocho de Lomatia hirsuta y Schinus patagonicus. El número de pies asignado a cada especie fue repartido uniformemente entre las diferentes clases diamétricas en nueve de los quince rodales, siguiendo a Clutter et al. (1983). Los árboles fueron cubicados según la fórmula de Smalian con longitud de troza igual o menor a un metro. En el caso de que el fuste presentara marcadas variaciones morfológicas (horquillas, fendas, cambios de eje central, etc.), la longitud de las trozas fue definida por la longitud de trozas de formas uniformes, siempre menores a $100 \mathrm{~cm}$.

El diámetro mínimo inventariable a $15 \mathrm{~cm}$ de altura de los árboles tipo se estableció en $3 \mathrm{~cm}$. Para cada árbol apeado se midió el DAP (diámetro normal a 1,3 $\mathrm{m}), \mathrm{D}$ (diámetro basal a $15 \mathrm{~cm}$ ), L (longitud de fuste hasta $2 \mathrm{~cm}$ de punta delgada), dos mediciones del diámetro de copa $\mathrm{D}_{\mathrm{C}}(\mathrm{cm})$ y $\mathrm{H}$ (altura total del árbol en metros). En lo sucesivo todas las referencias al diámetro, área basimétrica o volumen se establecerán en base a la sección basal.

La totalidad de las variables definidas anteriormente fueron utilizadas como variables independientes para análisis de regresión múltiple (método por pasos; probabilidad de $\mathrm{F}$ para entrar $\leq 0,05$; probabilidad de $\mathrm{F}$ para salir $\geq 0,05)$, tomando como variable dependiente el volumen $\left(\mathrm{V}: \mathrm{m}^{3}\right)$. Por su interés silvícola se calcularon además mediante regresión lineal (o linealizable) simple las relaciones dendrométricas: $\mathrm{DAP}=\mathrm{f}(\mathrm{D}), \mathrm{H}=\mathrm{f}(\mathrm{DAP})$ (cuadro 1).

La validez de los modelos de regresión fue verificada a través de ANOVA, habiendo analizado los supuestos de normalidad y homocedasticidad correspondientes.
Caracterización silvícola. Todas las parcelas fueron caracterizadas considerando el diámetro a $15 \mathrm{~cm}$ de altura (diámetro basal D $(\mathrm{cm})$ ) de todos los pies y especies presentes (cuadro 2). De la misma forma se definió la densidad ( $\mathrm{N}$ : pies ha ${ }^{-1}$ ), el área basimétrica a $15 \mathrm{~cm}$ de altura por hectárea (AB: $\mathrm{m}^{2} \mathrm{ha}^{-1}$ ) y el volumen por hectárea $\left(\mathrm{V}: \mathrm{m}^{3} \mathrm{ha}^{-1}\right)$ mediante las relaciones dendrométricas anteriormente comentadas. Como diámetro mínimo inventariable para la caracterización silvícola se tomaron cinco centímetros. Cada pie inventariable fue considerado como individuo independiente clasificándolo categóricamente como procedente de brote de cepa en caso de que fuera reconocible como tal. La altura dominante $\mathrm{H}_{0}$ (m) por parcela fue establecida midiendo la altura de los cuatro pies de mayor diámetro.

Para posibilitar una detallada descripción de la distribución de los tamaños de los árboles a nivel rodal se analizaron las variables estructurales relativas a la distribución diamétrica, densidad $\left(\mathrm{N}\right.$ : pies $\left.\mathrm{ha}^{-1}\right)$ y área basimétrica $\left(\mathrm{AB}: \mathrm{m}^{2} \mathrm{ha}^{-1}\right)$ de forma independiente. Así, se establecieron tres rangos: madera fina (D $\leq 15 \mathrm{~cm})$, madera media $(15,1 \mathrm{~cm} \leq \mathrm{D} \leq 30 \mathrm{~cm})$, madera gruesa (D > 30,1 cm), y se definieron las variables: $\mathrm{MF}=$ valor relativo del número de pies ha ${ }^{-1}$ de madera fina; $\mathrm{MM}=$ valor relativo del número de pies ha ${ }^{-1}$ de madera media; $\mathrm{MG}=$ valor relativo del número de pies ha ${ }^{-1}$ de madera gruesa; $\mathrm{ABMF}=$ valor relativo del área basimétrica total ocupada por pies de madera fina; $\mathrm{ABMM}=$ valor relativo del área basimétrica total ocupada por pies con diámetros de madera media; $\mathrm{ABMG}=$ valor relativo del área basimétrica total ocupada por pies de madera gruesa (cuadro 2). Para la especie principal, N. antarcti$c a$, se analizó la distribución diamétrica, calculando la distribución porcentual del área basimétrica y densidad ocupada por cada rango diametral (cuadro 2).

La composición específica a nivel de rodal fue evaluada definiendo los valores relativos en número de pies (N) y área basimétrica ( $\mathrm{AB}$ ) correspondientes a cada especie con respecto a los valores totales de ambas variables por parcela incluyendo detrás del acrónimo $(\mathrm{N} ; \mathrm{AB})$ las iniciales del nombre científico de la especie. Como especie dominante se definió a aquella que presentara valores relativos superiores al 50\%, tanto en densidad como en área basimétrica.

Para caracterizar cuantitativamente cada tipo estructural definido por Rusch (2005) se calcularon la media y los valores extremos de las principales variables dasométricas y dendrométricas del tipo de masa (bosque de ñire cerrado (BÑC), bosque de ñire semiabierto (BÑS), bosque mixto cerrado (BMC) y bosque mixto semiabierto (BMS)) para su presentación en forma de ficha descriptiva de grupo.

Por último, para explicar la variabilidad existente en los ñirantales se realizó un análisis factorial (extracción: componentes principales; criterio de extracción: autovalores > 1; método de rotación: Varimax normalizado) 
Cuadro 1. Parámetros y estadísticos para los modelos locales de las relaciones dendrométricas. Donde: $\mathrm{V}\left(\mathrm{m}^{3}\right)=\mathrm{volumen}$ total con corteza, DAP $(\mathrm{cm})$ = diámetro a la altura del pecho, $\mathrm{H}(\mathrm{m})$ = altura del árbol, D $(\mathrm{cm})$ = diámetro basal (altura de medición, $15 \mathrm{~cm})$. Se incluye en paréntesis el error típico corregido de cada parámetro.

Parameters and estimates for the local dendrometric equations. Where: $\mathrm{V}\left(\mathrm{m}^{3}\right)=$ over bark volume model, DAP $(\mathrm{cm})=$ diameter at breast height, $\mathrm{H}(\mathrm{m})$ = individual total height, $\mathrm{D}(\mathrm{cm})=$ basal diameter (height of measure, $15 \mathrm{~cm}$ ). Standard error of the estimates included in brackets.

\begin{tabular}{|c|c|c|c|c|c|c|}
\hline Especie & Variable dependiente & & Función & $P^{a}$ & $\mathrm{R}^{2}$ ajustado & $\mathrm{n}$ \\
\hline \multirow[t]{3}{*}{ Diostea juncea } & $\mathrm{V}\left(\mathrm{m}^{3}\right)$ & $\begin{array}{c}-0,0314 \\
(0,006)\end{array}$ & $\begin{array}{c}0,0057 * \text { DAP } \\
(0,00006)\end{array}$ & $* * *$ & 0,91 & 10 \\
\hline & DAP $(\mathrm{cm})$ & $\begin{array}{r}0,581 \\
(1,28)\end{array}$ & $\begin{array}{c}0,822 * \mathrm{D} \\
(0,1)\end{array}$ & $* * *$ & 0,88 & 10 \\
\hline & $\mathrm{H}(\mathrm{m})$ & $\begin{array}{c}3,183 \\
(0,87)\end{array}$ & $\begin{array}{c}0,31 * \text { DAP } \\
(0,08)\end{array}$ & $* *$ & 0,62 & 10 \\
\hline \multirow[t]{3}{*}{ Lomatia hirsuta } & $\mathrm{V}\left(\mathrm{m}^{3}\right)$ & $\begin{array}{c}-0,0467 \\
(0,01)\end{array}$ & $\begin{array}{c}0,0077 * \text { DAP } \\
(0,0008)\end{array}$ & $* * *$ & 0,93 & 8 \\
\hline & DAP $(\mathrm{cm})$ & $\begin{array}{c}(0,201 \\
(0,02)\end{array}$ & $\begin{array}{c}+0,007 * \mathrm{D})^{-1} \\
(0,001)\end{array}$ & $* *$ & 0,77 & 8 \\
\hline & $\mathrm{H}(\mathrm{m})$ & $\begin{array}{r}4,699 \\
(0,66)\end{array}$ & $\begin{array}{c}+0,142 * \text { DAP } \\
(0,046)\end{array}$ & $*$ & 0,55 & 8 \\
\hline \multirow[t]{3}{*}{ Nothofagus antarctica } & $\mathrm{V}\left(\mathrm{m}^{3}\right)$ & $\begin{array}{c}-0,059 \\
(0,013)\end{array}$ & $\begin{array}{c}+0,0088 * \text { DAP } \\
(0,0008)\end{array}$ & $* * *$ & 0,92 & 10 \\
\hline & DAP $(\mathrm{cm})$ & $\begin{array}{c}2,644 \\
(1,19)\end{array}$ & $\begin{array}{c}0,644 * \mathrm{D} \\
(0,07)\end{array}$ & $* * *$ & 0,92 & 10 \\
\hline & $\mathrm{H}(\mathrm{m})$ & $\begin{array}{c}3,277 \\
(1,2)\end{array}$ & $\begin{array}{c}+0,278 * \mathrm{DAP} \\
(0,08)\end{array}$ & $* *$ & 0,55 & 10 \\
\hline \multirow[t]{3}{*}{ Schinus patagonicus } & $\mathrm{V}\left(\mathrm{m}^{3}\right)$ & $\begin{array}{c}(0,0106 \\
(0,013)\end{array}$ & $\begin{array}{c}0,0135 * \mathrm{DAP})^{2} \\
(0,0016)\end{array}$ & $* * *$ & 0,91 & 8 \\
\hline & $\mathrm{DAP}(\mathrm{cm})$ & $\begin{array}{c}-0,524 \\
(1,08)\end{array}$ & $\begin{array}{c}0,862 * \mathrm{D} \\
(0,106)\end{array}$ & $* * *$ & 0,90 & 8 \\
\hline & $\mathrm{H}(\mathrm{m})$ & $\begin{array}{c}3,236 \\
(0,65)\end{array}$ & $\begin{array}{c}0,195 * \mathrm{DAP} \\
(0,19)\end{array}$ & $*$ & 0,44 & 8 \\
\hline
\end{tabular}

$\mathrm{a} *=P<0,05 ; * *=P<0,01 ; * * *=P<0,001$.

sobre la totalidad de parcelas y las variables: $\mathrm{N}, \mathrm{AB}, \mathrm{D}$, D (N.a.), $\mathrm{H}_{0}, \mathrm{~N}(\%$ N.a. $), \mathrm{AB}(\%$ N.a. $), \mathrm{MF}, \mathrm{MM}, \mathrm{MG}$, ABMF, ABMM, ABMG. No se incluyeron en el análisis las variables relativas al volumen para evitar la acumulación de los errores propios de las ecuaciones dendrométricas.

Clave tipológica de ñirantales. Como herramienta de clasificación se realizó una clave tipológica de clasificación binaria y dicotómica buscando establecer los valores de las variables que definieran la máxima variabilidad entre subgrupos. El método de partición recursiva no paramétrica y no lineal fue CART (classification and regression trees) con búsqueda exhaustiva de particiones univariadas (norma de parada: parámetro de parada $=0,1$ fracción del objeto; potencial para selección de variable de partición $=0,05$; bondad de ajuste: medida Gini). El establecimiento de la clave dicotómica puede verse negativamente alterado por el efecto reiterativo de variables correlacionadas entre sí, siendo recomendable incluir en el análisis una sola variable de cada par altamente correlacionado. Es por ello que como fase previa a la definición de la clave tipológica se calculó la matriz de correlaciones de Spearman. Para evitar la incidencia que podrían ocasionar las variables representativas de la distribución diamétrica (densidad $(\mathrm{N})$ y área basimétrica 
(AB)) se seleccionaron para el análisis de clasificación únicamente las variables asociadas a la densidad en el caso de que el coeficiente de correlación de Spearman entre $\mathrm{N}$ y $\mathrm{AB}$ fuera mayor que 0,7 .

\section{RESULTADOS}

Los modelos dendrométricos desarrollados para estimar el volumen individual, a partir del diámetro normal (DAP), y el DAP muestran en general coeficientes de determinación superiores a 0,9 (cuadro 1). En contraposición, los menores ajustes se observaron en los modelos desarrollados para estimar la altura $(\mathrm{H})$ a partir del DAP.

La distribución diamétrica de los ñirantales indica que, tanto en número de pies por hectárea como en área basimétrica, la mayor representación correspondió a pies categorizados como de madera media $(\mathrm{MM}=50 \%$; $\mathrm{ABMM}=60 \%$ ) (cuadro 2). Únicamente seis variables (NN.a., ABN.a., DmN.a., $\mathrm{H}_{0}$, MF, MM) presentaron una distribución ajustada a la normalidad, apareciendo una distribución bimodal de algunas variables entre las que destaca el área basimétrica total (figura 1). También resalta la elevada desviación estándar de la densidad $(\mathrm{N})$ o del área basimétrica $(\mathrm{AB})$, mostrando una marcada variabilidad y heterogeneidad espacial entre rodales en la zona de estudio.

Nothofagus antarctica apareció como especie dominante en la zona de estudio al ocupar más del $50 \%$ de la densidad total y del área basimétrica total (cuadro 2). Dentro de las especies acompañantes, las dos especies con mayor contribución en la densidad total fueron $S$. patagonicus (18\%) y D. juncea (16\%), seguidos de L. hirsuta $(9,6 \%)$. La presencia de chacay (Discaria serratifolia (Vent.) Mart.) y de M. boaria fue muy reducida, tomando en todos los tipos de masa valores inferiores al 3\% (cuadro 2).

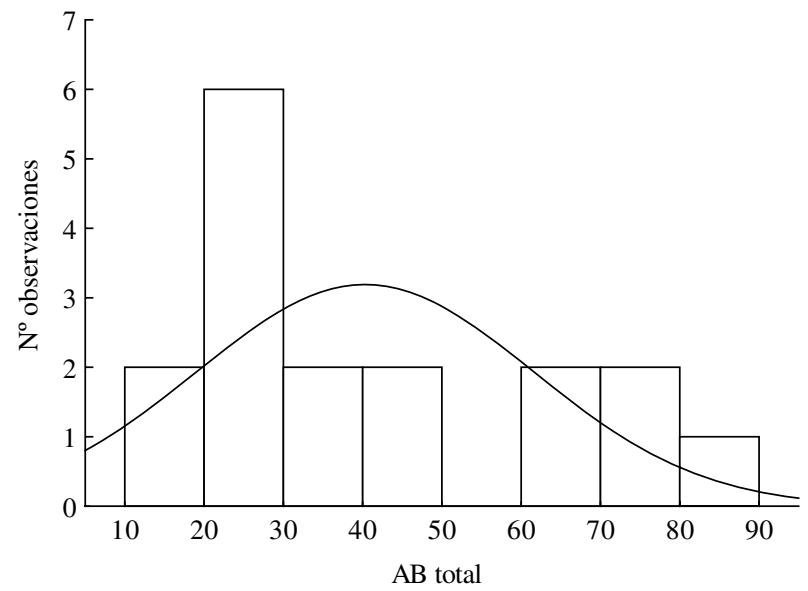

Figura 1. Histograma de frecuencias y frecuencia normal esperada de la variable área basimétrica total (ABtotal: $\mathrm{m}^{2} \mathrm{ha}^{-1}$ ).

Frequency histogram of the total basal area (ABtotal:
Cuadro 2. Variables descriptivas medias de las masas forestales de la cuenca central del Río Foyel (Provincia de Río Negro; Patagonia Argentina). Donde: AB (\%N.a.) = valor relativo del área basimétrica total ocupada por $N$. antarctica; $\mathrm{ABMF}$ : valor relativo del área basimétrica total ocupada por pies con diámetros $\leq 15 \mathrm{~cm}$; ABMG: valor relativo del área basimétrica total ocupada por pies con diámetros mayores a $30,1 \mathrm{~cm}$; ABMM: valor relativo del área basimétrica total ocupada por pies con diámetros situados en el rango $15,1 \mathrm{~cm} \leq$ Diámetro basal $\leq 30 \mathrm{~cm}$; ABtotal = área basimétrica $\left(\mathrm{m}^{2} \mathrm{ha}^{-1}\right)$ total; Dm $($ total $)=$ diámetro medio $(\mathrm{cm})$ de todos los pies por ha; Dm (N.a.) = diámetro medio $(\mathrm{cm})$ de todos los pies de $N$. antarctica por ha; $\mathrm{H}_{\mathrm{O}}=$ altura dominante $(\mathrm{m})$; MF: valor relativo del número de pies/ha con diámetros $\leq 15 \mathrm{~cm}$; MG: valor relativo del número de pies/ha con diámetros > 30,1 cm; MM: valor relativo del número de pies/ha con diámetros situados en el rango $15,1 \mathrm{~cm} \leq$ Diámetro basal $\leq 30 \mathrm{~cm} ; \mathrm{N} \% \mathrm{~N}$.a. = valor relativo del número de pies por hectárea de Nothofagus antarctica; Ntotal $=$ densidad total (número total de pies por hectárea $) ;$ Vol = volumen $\left(\mathrm{m}^{3} \mathrm{ha}^{-1}\right) ;$ D.j. = Diostea juncea ; D.s. = Discaria serratifolia; L.h. = Lomatia hirsuta; N.a. = Nothofagus antarctica $;$ S.p. = Schinus patagonicus

Mean values of descriptive variables of the stands of the central basin of the Foyel River (Río Negro province; Patagonia Argentina). Where: $\mathrm{AB}(\% \mathrm{~N} . \mathrm{a})=$. proportion of the total basal area corresponding to $N$. antarctica; ABMF: relative values of ABtotal of trees with diameters $\leq 15 \mathrm{~cm}$; ABMG: relative values of ABtotal of trees with diameters $>30,1 \mathrm{~cm}$; ABMM: relative values of ABtotal of trees with diameters in the range $15,1 \mathrm{~cm} \leq$ diameter $\leq 30 \mathrm{~cm}$; ABtotal $=$ total basal area $\left(\mathrm{m}^{2} \mathrm{ha}^{-1}\right) ; \mathrm{Dm}($ total $)=$ total average diameter $(\mathrm{cm})$ per ha; Dm (N.a.) = total average diameter $(\mathrm{cm})$ per ha of $N$. antarctica; $\mathrm{H}_{\mathrm{O}}=$ dominant height (m); MF: proportion of trees per ha with diameters $\leq 15 \mathrm{~cm}$; MG: proportion of trees per ha with diameters $>30,1 \mathrm{~cm}$; MM: proportion of trees per ha with diameters in the range $15,1 \mathrm{~cm} \leq$ diameter $\leq 30 \mathrm{~cm}$; N\%N.a. = proportion of trees per ha of Nothofagus antarctica; Ntotal = total density (number of trees per ha); Vol $=$ volumen $\left(\mathrm{m}^{3} \mathrm{ha}^{-1}\right) ; \mathrm{D} . \mathrm{j} .=$ Diostea juncea; D.s. = Discaria serratifolia $;$ L.h. = Lomatia hirsuta $;$ N.a. = Nothofagus antarctica; S.p. = Schinus patagonicus.

\begin{tabular}{lccccc}
\hline & \multicolumn{2}{c}{ Media $\pm \mathrm{S}$} & Mínimo & Máximo & $P^{\mathrm{a}}$ \\
\hline AB(\%N.a.) & $64,7 \pm$ & 31,6 & 6,6 & 100 & n.s. \\
ABMF(\%) & $0,1 \pm$ & 0,19 & - & 0,7 & $* *$ \\
ABMG(\%) & $0,2 \pm$ & 0,33 & - & 1 & $* * *$ \\
ABMM(\%) & $0,59 \pm$ & 0,27 & - & 0,8 & $* *$ \\
ABtotal & $40,3 \pm$ & 21,26 & 17,3 & 80,7 & $*$ \\
Dm (total) & $15,4 \pm$ & 5,41 & 10 & 31,9 & $* *$ \\
Dm(N.a.) & $18,9 \pm$ & 6,74 & 10,7 & 34,2 & n.s. \\
Ho & $9,5 \pm$ & 3,09 & 4,5 & 17 & n.s. \\
MF(\%) & $0,3 \pm$ & 0,26 & 0 & 0,9 & n.s. \\
MG(\%) & $0,15 \pm$ & 0,31 & 0 & 1 & $* * *$ \\
MM(\%) & $0,49 \pm$ & 0,25 & 0 & 0,9 & n.s. \\
N(\%N.a.) & $52,4 \pm$ & 33,59 & 1,9 & 100 & n.s. \\
Ntotal & 2.470 & $\pm 1.958,82$ & 240 & 6.781 & $*$ \\
Vol(D.j.) & $14,7 \pm$ & 26,29 & 0,00 & 101,07 & $* * *$ \\
Vol(L.h.) & $7,54 \pm$ & 13,95 & 0,00 & 55,30 & $* * *$ \\
Vol(N.a.) & $72,3 \pm$ & 66,2 & 6,3 & 247,9 & $* *$ \\
Vol(S.p.) & $5,21 \pm$ & 11,68 & 0,00 & 49,42 & $* * *$ \\
\hline
\end{tabular}

a Potencial de la prueba de normalidad de Shapiro-Wilk.

$*=P<0,05 ; * *=P<0,01 ; * * *=P<0,001 ; \mathrm{ns}=$ no significativo. $\left.\mathrm{m}^{2} \mathrm{ha}^{-1}\right)$ 
La ficha descriptiva de los diferentes tipos de masa (cuadros 3 y 4) mostró las mayores densidades (Ntotal) en los tipos de masa clasificados como bosque de ñire cerrado (BÑC) y bosque de ñire semiabierto (BÑS). También se presentaron en estos tipos de masa los mayores porcentajes de pies clasificados como de madera fina (figura 2). Las distribuciones diamétricas indican en todos los casos una forma principal de masa regular al incluirse la mayoría de pies dentro de una clase diametral. En todos los casos, menos en BÑC, la regularidad es motivada por la preponderante presencia de pies asignados a la clase madera media (MM). En el tipo de masa bosque de ñire cerrado (BÑC) la forma principal de masa puede clasificarse como estructuralmente coetánea al encuadrarse mayoritariamente los pies en un rango diametral reducido (figura 2). El mayor número de pies clasificados como originados de brote de cepa se presentó en el tipo de masa BÑC $(33,5 \%)$ seguido del BMS $(8,6 \%)$ y BÑS $(6,4 \%)$. En el tipo BMC no se reconocieron pies procedentes de brote de cepa.

Cuadro 3. Ficha descriptiva y variables dasométricas básicas de los cuatro tipos estructurales de ñirantales. Acrónimos en cuadro 2 .

Descriptive files and dasometric variables of the four structural types of "ñirantales". Acronisms in table 2.

\begin{tabular}{|c|c|c|c|c|c|c|c|c|c|c|c|c|}
\hline \multirow[t]{2}{*}{ Variable } & \multicolumn{3}{|c|}{$\begin{array}{l}\text { Bosque mixto cerrado } \\
\qquad \begin{array}{c}(\mathrm{BMC}) \\
(\mathrm{n}=5)\end{array}\end{array}$} & \multicolumn{3}{|c|}{$\begin{array}{l}\text { Bosque de ñire cerrado } \\
(\text { BÑC) } \\
(\mathrm{n}=2)\end{array}$} & \multicolumn{3}{|c|}{$\begin{array}{l}\text { Bosque mixto semiabierto } \\
\qquad \begin{array}{c}(\mathrm{BMS}) \\
(\mathrm{n}=5)\end{array}\end{array}$} & \multicolumn{3}{|c|}{$\begin{array}{l}\text { Bosque de ñire semiabierto } \\
\qquad \begin{array}{c}(\mathrm{BN} S) \\
(\mathrm{n}=5)\end{array}\end{array}$} \\
\hline & Media & Mínimo & Máximo & Media & Mínimo & Máximo & Media & Mínimo & Máximo & Media & Mínimo & Máximo \\
\hline $\mathrm{AB}$ & 57 & 37,8 & 72 & 71,3 & 61,8 & 80,7 & 26,8 & 22 & 37,4 & 24,7 & 17,3 & 43,3 \\
\hline $\mathrm{Ab}(\%$ N.a. $)$ & 35,9 & 6,6 & 65,8 & 99,9 & 99,8 & 100 & 51,2 & 27 & 78,9 & 93,2 & 81,5 & 99,6 \\
\hline Dm (total) & 13,6 & 10 & 18,6 & 12,5 & 10,7 & 14,2 & 14,9 & 10,8 & 23,4 & 19,1 & 14,3 & 31,9 \\
\hline Dm(N.a.) & 20,9 & 11,3 & 30 & 12,5 & 10,7 & 14,2 & 16,8 & 13 & 24,9 & 21,5 & 15 & 34,2 \\
\hline Ho & 10,5 & 9,7 & 12,5 & 5,5 & 5,5 & 5,5 & 7,6 & 4,5 & 10,5 & 12,16 & 10 & 17 \\
\hline N\%N.a. & 17,6 & 1,9 & 34 & 99,6 & 99,2 & 100 & 41 & 20 & 60,7 & 80 & 59,5 & 98 \\
\hline NN.a. & 597,2 & 40 & 1.080 & 5.450 & 4.700 & 6.200 & 604 & 260 & 1240 & 752 & 220 & 1.320 \\
\hline Ntotal & $3.580,2$ & 1.760 & 6.781 & 5.475 & 4.700 & 6.250 & 1.688 & 440 & 3.080 & 940 & 240 & 1.640 \\
\hline Vol(D.j.) & 32,2 & 4,6 & 101,1 & 0 & 0 & 0 & 15,5 & 0,00 & 46,4 & 2,3 & 0,00 & 8,6 \\
\hline Vol(L.h.) & 11,2 & 0 & 22 & 0 & 0 & 0 & 14,5 & 0,00 & 55,3 & 0,00 & 0,00 & 0,00 \\
\hline Vol(N.a.) & 48,6 & 6,3 & 109,4 & 223,6 & 199 & 247,9 & 40,5 & 21,1 & 71,1 & 67,2 & 44,23 & 119,6 \\
\hline Vol(S.p.) & 13 & 1,4 & 49,4 & 0 & 0 & 0 & 3,1 & 0 & 5,4 & 1,6 & 0,23 & 3,4 \\
\hline
\end{tabular}

Cuadro 4. Dominancia (\%) de especies en los cuatro tipos estructurales de ñirantales.

Dominance of species in the four structural types of "ñirantales" (BMC $=$ closed mixed forest, BÑC $=$ closed ñre forest, $\mathrm{BMS}=$ semi-open mixed forest, $\mathrm{BN} S=$ semi-open ñire forest).

\begin{tabular}{|c|c|c|c|c|c|c|c|c|c|c|c|c|}
\hline \multirow[t]{2}{*}{ Variable } & \multicolumn{3}{|c|}{$\begin{array}{l}\text { Bosque mixto cerrado } \\
\qquad \begin{array}{l}(\mathrm{BMC}) \\
(\mathrm{n}=5)\end{array}\end{array}$} & \multicolumn{3}{|c|}{$\begin{array}{l}\text { Bosque de ñire cerrado } \\
\qquad \begin{array}{c}(\mathrm{BN} C) \\
(\mathrm{n}=2)\end{array}\end{array}$} & \multicolumn{3}{|c|}{$\begin{array}{l}\text { Bosque mixto semiabierto } \\
\qquad \begin{array}{c}(\mathrm{BMS}) \\
(\mathrm{n}=5)\end{array}\end{array}$} & \multicolumn{3}{|c|}{$\begin{array}{l}\text { Bosque de ñire semiabierto } \\
\qquad \begin{array}{c}(\mathrm{BN} S) \\
(\mathrm{n}=5)\end{array}\end{array}$} \\
\hline & Media & Mínimo & Máximo & Media & Mínimo & Máximo & Media & Mínimo & Máximo & Media & Mínimo & Máximo \\
\hline $\begin{array}{l}\text { Diostea } \\
\text { juncea }\end{array}$ & 0,3 & 0,04 & 0,8 & 0 & 0 & 0 & 0,2 & 0 & 0,7 & 0,01 & 0 & 0,02 \\
\hline Discaria & & & & & & & & & & & & \\
\hline serratifolia & 0 & 0 & 0,02 & 0,004 & 0 & 0,008 & 0,04 & 0 & 0,2 & 0,02 & 0 & 0,08 \\
\hline $\begin{array}{l}\text { Lomatia } \\
\quad \text { hirsuta }\end{array}$ & 0,2 & 0 & 0,4 & 0 & 0 & 0 & 0,2 & 0 & 0,6 & 0 & 0 & 0 \\
\hline $\begin{array}{r}\text { Maytenus } \\
\text { boaria }\end{array}$ & 0,01 & 0 & 0,05 & 0 & 0 & 0 & 0,03 & 0 & 0,1 & 0 & 0 & 0 \\
\hline $\begin{array}{l}\text { Nothofagus } \\
\text { antarctica }\end{array}$ & 0,2 & 0,02 & 0,3 & 1 & 0,9 & 1 & 0,4 & 0,2 & 0,6 & 0,8 & 0,6 & 0,9 \\
\hline $\begin{array}{l}\text { Schinus } \\
\quad \text { patagonicus }\end{array}$ & $s \quad 0,4$ & 0,07 & 0,6 & 0 & 0 & 0 & 0,1 & 0 & 0,2 & 0,1 & 0 & 0,3 \\
\hline
\end{tabular}




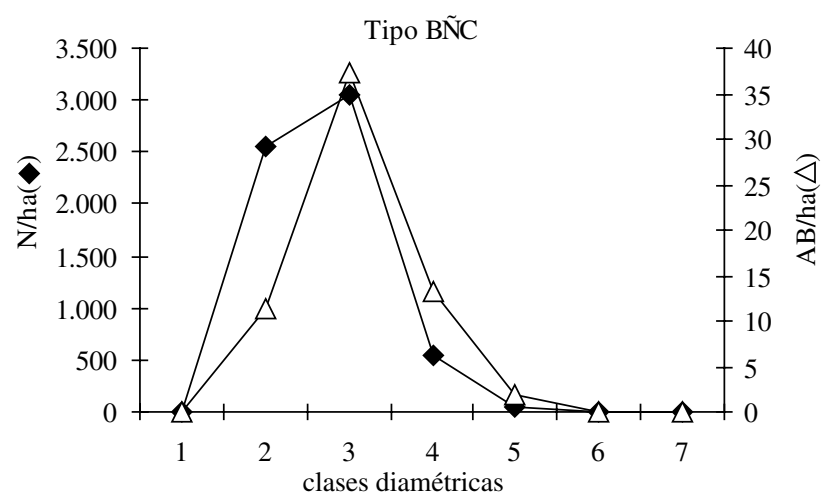

TIPO BMC

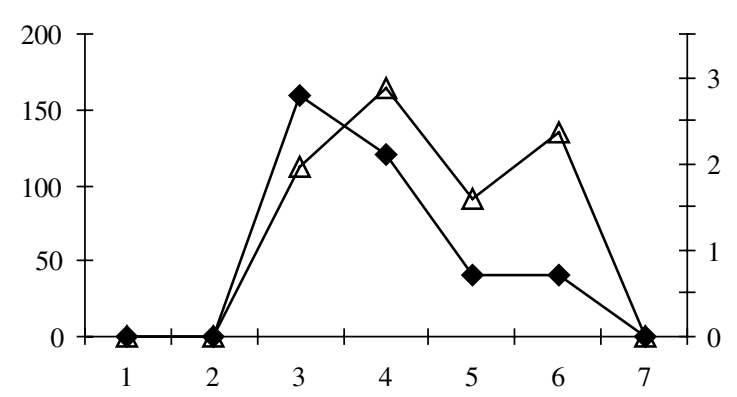

Tipo BÑS

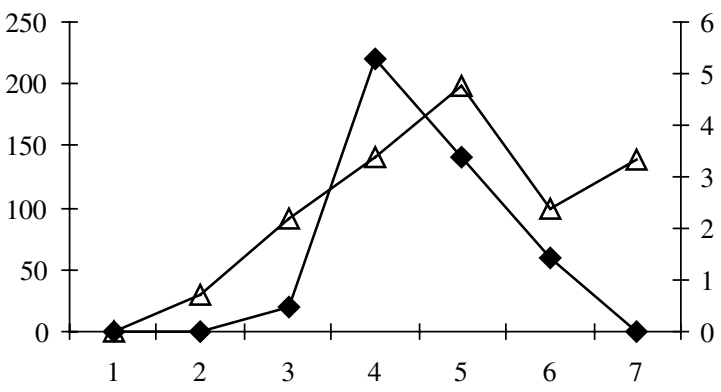

Tipo BMS

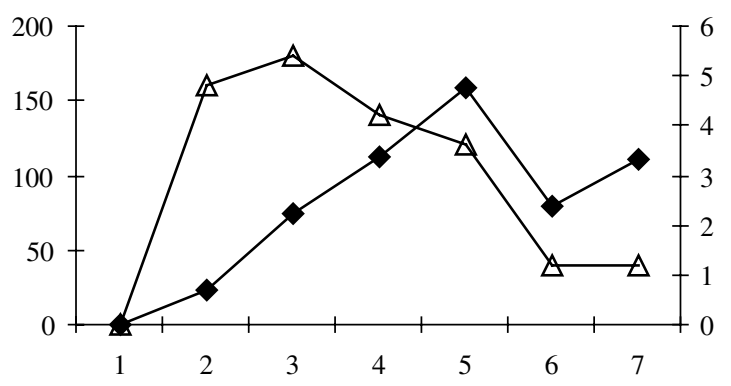

Figura 2. Distribución diamétrica de la densidad $\left(\mathrm{N} \mathrm{ha}^{-1}\right)$ y el área basimétrica $\left(\mathrm{AB}: \mathrm{m}^{2} \mathrm{ha}^{-1}\right)$ de los cuatro tipos estructurales de ñirantales. Eje de abscisas: clases diamétricas; eje de ordenadas izquierdo: densidad $\left(\mathrm{N} \mathrm{ha}^{-1}\right)$; eje de ordenadas derecho: área basimétrica por hectárea $\left(\mathrm{AB} \mathrm{ha}^{-1}\right)$. Acrónimos en cuadros 3 y 4.

Diametric distribution of density $\left(\mathrm{N} \mathrm{ha}^{-1}\right)$ and basal area $\left(\mathrm{AB}: \mathrm{m}^{2} \mathrm{ha}^{-1}\right)$ of the four structural types of "ñirantales". Abscissa axis: diametric classes; left ordinate axis: density $\left(\mathrm{N} \mathrm{ha}^{-1}\right)$; right ordinate axis: basal area per hectare $\left(\mathrm{AB} \mathrm{ha}{ }^{-1}\right)$. Acronims in tables 3 and 4 .

Los cuatro primeros ejes (autovalores $>1$ ) del análisis de componentes principales explicaron el $94 \%$ de la variabilidad existente, presentándose el primer eje fuertemente saturado por valores asociados a pies de grandes dimensiones (cuadro 5, figura 3). Podría interpretarse este eje como una representación del estado de desarrollo al aparecer en el semieje positivo variables relativas a la altura dominante $\left(\mathrm{H}_{0}\right)$, diámetros medios elevados $(\mathrm{Dm})$ o la presencia de pies de madera gruesa (MG, ABMG). Estos valores se contraponen en el semieje negativo con variables representativas de pies de pequeñas dimensiones (MF (\%), ABMF (\%)). En el mismo sentido, en el segundo eje se confrontaron valores asociados a pies de mediano desarrollo con pies de grandes desarrollos. El tercer eje puede explicarse como indicador de la dominancia relativa en densidad y área basimétrica de $N$. antarctica al presentar las variables asociadas a la dominancia del ñire $(\mathrm{N} \% \mathrm{Na} ; \mathrm{AB} \% \mathrm{Na})$ valores próximos a la unidad. Las variables con ponderaciones altas en el cuarto factor aluden a la espesura. Así, se agruparon con cargas altas en el entorno positivo del eje las variables área basimétrica total $(\mathrm{AB})$ y la densidad total $(\mathrm{N})$.
La clave tipológica de clasificación, con una eficacia en la validación cruzada del $100 \%$, presentó tres particiones y cuatro puntos terminales (figura 4). Las constantes que definen las particiones describen los mismos factores que los comentados en el análisis de componentes principales. Así, como primera variable de partición apareció la dominancia de $N$. antarctica representada a través del área basimétrica ocupada por ñire $(\mathrm{AB}(\% \mathrm{NA})<80 \%)$. La espesura, manifestada a través del área basimétrica total $\left(\mathrm{AB}<37,5 \mathrm{~m}^{2} \mathrm{ha}^{-1}\right)$, y el estado de desarrollo, expresado mediante la altura dominante $\left(\mathrm{H}_{0}<7,75 \mathrm{~m}\right)$, aparecieron como segunda y tercera constantes de partición.

\section{DISCUSIÓN Y CONCLUSIONES}

Los ñirantales de la cuenca del río Foyel pueden adscribirse al morfotipo arborescente descrito por Ramírez et al. (1985). Considerando su porte arbóreo (altura dominante media $\left.\mathrm{H}_{0}=9,6 \mathrm{~m}\right)$ y diámetro $(\mathrm{D}=18,9 \mathrm{~cm})$ permiten ser clasificados como ecosistemas boscosos atendiendo a su altura (SECF 2005). Estos ecosistemas fores- 
Cuadro 5. Matriz de componentes rotados en el análisis factorial de la totalidad de parcelas de inventario (método de extracción: componentes principales; método de rotación: Varimax normalizado). Únicamente se presentan saturaciones mayores a [0,4]. Acrónimos en cuadro 2.

Matrix of rotated components of the factorial analysis for the studied plots (extraction method: principal components; rotation method: normalized Varimax). Only saturation values higher than [0.4] are presented. Acronisms in table 2.

\begin{tabular}{lrrrr}
\hline & \multicolumn{4}{c}{ Componente } \\
\cline { 2 - 5 } & 1 & 2 & 3 & 4 \\
\hline MF(\%) & $-0,95$ & - & - & - \\
ABMF(\%) & $-0,91$ & - & - & - \\
Ho & 0,88 & - & - & - \\
Dm(N.a.) & 0,84 & 0,46 & - & - \\
Dm (total) & 0,73 & - & 0,41 & - \\
ABMM(\%) & - & $-0,95$ & - & - \\
MM(\%) & - & $-0,91$ & - & - \\
MG(\%) & 0,58 & 0,78 & - & - \\
ABMG(\%) & 0,68 & 0,70 & - & - \\
N(\%N.a.) & - & - & 0,98 & - \\
Ab(\%N.a.) & - & - & 0,97 & - \\
ABTOTAL & - & - & - & 0,97 \\
NTOTAL & $-0,50$ & - & - & 0,82 \\
$\%$ varianza explicada & 47,43 & 21,42 & 16,73 & 8,60 \\
$\%$ acumulativo & 47,43 & 68,85 & 85,58 & 94,18 \\
\hline
\end{tabular}

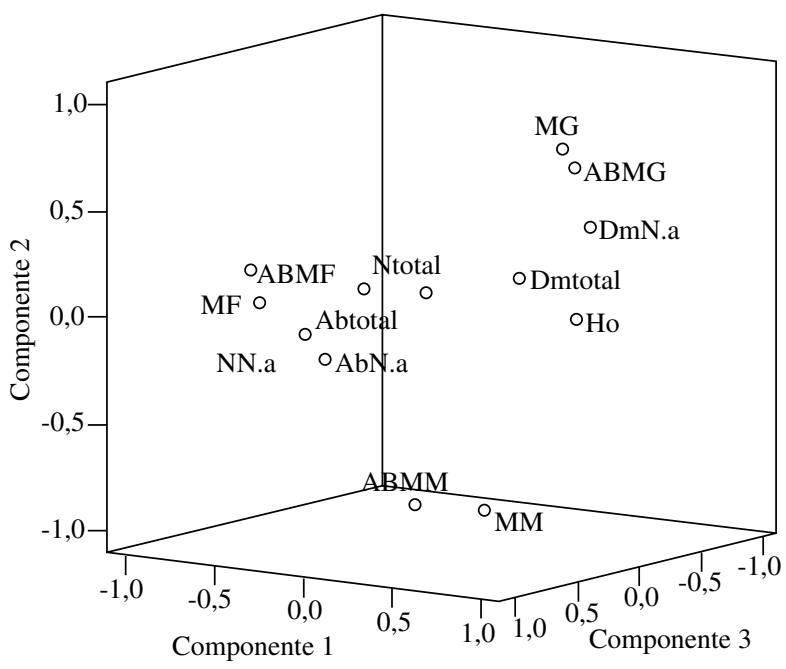

Figura 3. Representación gráfica y en tres dimensiones de los tres primeros ejes del análisis factorial. Acrónimos en cuadro 2.

Three dimensional representation of the first three axis $\left(\mathrm{C}_{\mathrm{i}}\right)$ of the factorial analysis. Acronisms in table 2.

tales presentan bajas existencias $\left(\mathrm{m}^{3} \mathrm{ha}^{-1}\right)$ en comparación con las masas forestales dominadas por $N$. antarctica descritas en otras zonas de la Patagonia o la Araucanía (Peri et al. 2005, Hansen et al. 2005, Pauchard et al. 2006) (cuadro 2).

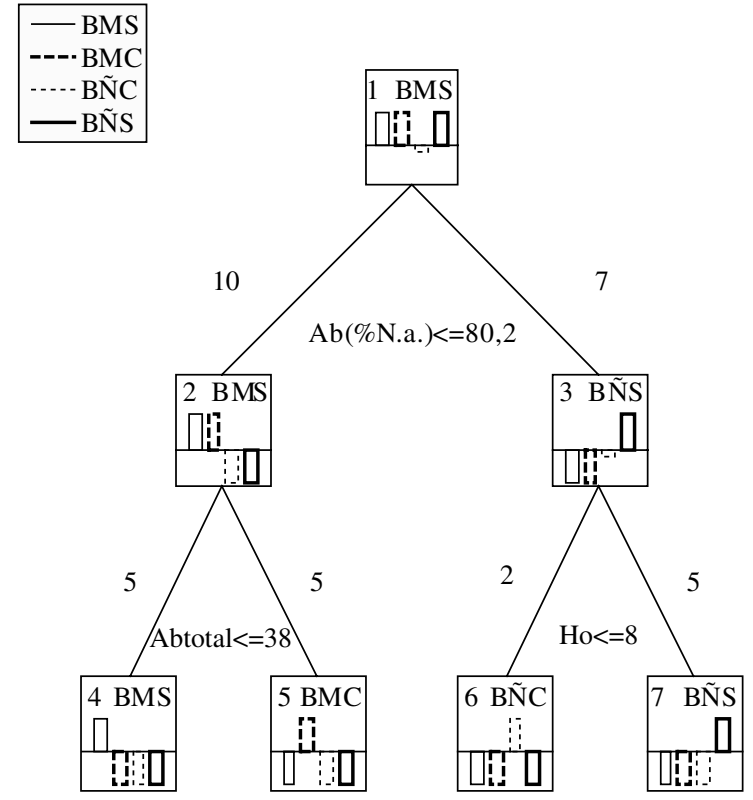

Figura 4. Clave dicotómica (árbol de clasificación), variables de partición y diagramas de desviaciones de los cuatro tipos estructurales de ñirantales. Acrónimos en cuadros 2 y 3 .

Dicotomic key (classification tree), split variables and deviation diagrams for the four structural types of "ñirantales". Acronisms en tables 2 and 3 .

El conjunto de ecuaciones dendrométricas definidas para su caracterización supone un avance importante para la estimación del rendimiento volumétrico de los ñirantales del noroeste patagónico. Sin embargo, para ajustar un modelo general por especie es necesario el desarrollo de modelos basados en un mayor número de árboles tipo apeados en diferentes estaciones ecológicas. Incluso deberían validarse modelos desarrollados en sitios distantes como los ñirantales de Tierra del Fuego (Martínez-Pastur et al. 1995). Cabe señalar que el presente estudio no consideró los ñirantales bajos (del tipo nanofanerofítico) por valorarse que su uso con fines maderables era prácticamente nulo y por poseer la mayor parte de sus plantas un diámetro basal inferior al mínimo inventariable.

Desde un punto de vista dasonómico, los ñirantales del río Foyel presentan en la actualidad y de manera mayoritaria una forma fundamental de masa de monte bajo (masa arbórea compuesta por pies cuyo origen es un brote de cepa o de raíz). Aparecen también rodales en monte medio, mostrando ambas estructuras antecedentes silvopastoriles ligados al uso antrópico del fuego y al aprovechamiento de leña. La fuerte variabilidad interrodal encontrada muestra una marcada afinidad con la mayoría de los montes bajos y medios estructurales de especies caducifolias de latitudes templadas. Como rasgo característico desde un punto de vista de la estratificación horizontal, las masas forestales de la zona de estudio son al mismo tiempo heterogéneas a nivel interno 
pero globalmente muy similares (Ducrey 1992, Dubourdieu 1997). Los análisis multivariantes (CART y Factorial) indican, en este sentido, que la variabilidad y la separación en grupos estructurales es explicable a través de la distribución diamétrica, la espesura y la composición específica, y muestran el interés silvícola de la clasificación tipológica y binaria basada en estas variables.

La marcada variabilidad interrodal puede entenderse atendiendo al estado de desarrollo intermedio en el que se encuentran estos ecosistemas forestales, a diferentes condiciones microestacionales y al tradicional uso silvopastoril. Destaca, no obstante, el volumen en pie de los bosques de ñire cerrado (BÑC) $\left(223 \mathrm{~m}^{3} \mathrm{ha}^{-1}\right)$, los cuales presentan una cierta similitud estructural con los ñirantales de condición hídrica intermedia analizados por Hansen et al. (2005) en la provincia de Chubut (Argentina) (cuadro 5). Atendiendo al estado de desarrollo en el que se encuentran estos tipos de masa (diámetro medio cuadrático: $\left.\mathrm{D}_{\mathrm{m}}=12,5 \mathrm{~cm}\right)$ y a las elevadas densidades $(\mathrm{N}>$ $4.500 \mathrm{~m}^{3} \mathrm{ha}^{-1}$ ) se abre la posibilidad de considerar la implementación de modelos silvícolas basados en el manejo de la espesura y la ocupación completa del potencial forestal productivo del sitio (Assmann 1961).

Dentro de las especies acompañantes resaltan las elevadas existencias de $D$. juncea en las estructuras de bosque mixto cerrado (BMC). Con $32 \mathrm{~m}^{3} \mathrm{ha}^{-1}$ el conocimiento de la fruticeticultura (gestión de matorrales y arbustedas (fruticetum)) de D. juncea se presenta como un interesante reto a abordar en la gestión forestal sostenible por su alta capacidad de reproducción vegetativa y poder calorífico.

En el análisis de las curvas de distribución diamétrica de $N$. antarctica (figura 2) llama la atención la inflexión que se produce en las clases diamétricas superiores (> $27,5 \mathrm{~cm}$ ). Este cambio de pendiente, especialmente marcado en las curvas del área basimétrica, muestra, como era de esperar, que la mayor contribución en el área basimétrica total es aportada por un número reducido de pies de grandes dimensiones. La presencia de una significativa representación de pies predominantes (con copas que alcanzan y forman el nivel máximo del vuelo y reciben luz en su parte superior) (SECF 2005) abre la opción a que las claras o raleos puedan plantearse como raleos mixtos o por lo alto, apeándose en este tratamiento pies del estrato superior con el objetivo de favorecer el desarrollo de las copas de árboles seleccionados por su calidad. Al mismo tiempo, se busca incrementar su valor agregado y se favorece la estabilidad del rodal (SECF 2005, SilvaVoc 2006). Se eliminan en este tratamiento todos aquellos pies que compitan directamente con los árboles dominantes de mejores características mantenidos en pie, aunque tengan dimensiones considerables y características morfológicas no demasiado negativas (Schütz 1990). Se respetarían también los pies de menor diámetro, con poca biomasa foliar, inclinados en mayor o menor grado, pensando que su escaso desarrollo actual impedirá tanto su respuesta a la disminución de competencia ocasionada por la corta como que compitan de forma significativa por luz con los pies seleccionados (resalvos). El mantenimiento del estrato dominado es valorado como positivo, pues actúa reduciendo en parte el rebrote con su presencia, aportando al mismo tiempo al fuste una cierta protección frente a los herbívoros (rascado, escodado, etc.).

Coincidiendo con Claps y Schorr (2005), el establecimiento de una silvicultura tendiente a obtener pies de mayor valor unitario destinados al uso como postes (frente al de leña) puede ser interpretada como una opción para mejorar la rentabilidad de los ñirantales. No se evalúan en el presente trabajo las producciones forrajeras asociadas a los diferentes tipos de masa. No obstante, la presencia de ganado en los rodales en estadios intermedios de desarrollo como los descritos puede ser considerada como positiva al poderse valorar como herramienta de control de rebrote y de prevención de incendios al evitar la acumulación de biomasa que puede favorecer la continuidad horizontal de combustible (Hubert 1983, Dubourdieu 1991). Sin embargo, es menester citar que ningún modelo de gestión forestal sostenible es compatible con la presencia de ganadería extensiva si no considera en alguna de sus etapas la implementación de eficaces medidas de control de las cargas animales tendientes a garantizar la regeneración natural.

Atendiendo a las características similares a las de la mayoría de montes bajos y medios estructurales de bosques caducifolios de climas templados (destacando los géneros Quercus y Fagus del hemisferio norte, evolutivamente emparentados con Nothofagus (Puntieri et al. 2006)): gran heterogeneidad interrodal pero uniformidad global (Lanier 1986, Dubourdieu 1997), los modelos silvícolas a plantear no deberían buscar la aplicación de modelos de gestión de masa basados en el seguimiento de normas de regulación o manejo estrictas ni en la consecución de una organización estructural o espacio-temporal determinada al partirse de un tipo de masa muy alejada de cualquier patrón de organización. Por ello, los tratamientos a aplicar se deberán ajustar a las necesidades silvícolas de cada estrato vertical y rodal y, dentro de éstos, a las de cada tipo de masa o bosquete (Ciancio et al. 1990, Donoso 1994).

Siendo uno de los objetivos más importantes de la gestión forestal sostenible el de conseguir y mantener continuadamente una masa forestal en óptimo estado vegetativo y con posibilidad real de regeneración sexual, el método de ordenación o modelo de gestión planteado se ajustaría al definido como Método Cultural o Método Silvícola (Pardé 1930).

El presente trabajo supone una primera aproximación metodológica para el conocimiento de la silvicultura de las masas nativas de Nothofagus antarctica del noroeste de la Patagonia. La clasificación dasométrica y clave tipológica deben ser entendidas como herramientas objeti- 
vas de análisis y descripción operacional de los ñirantales. Como útiles de inventario tipológico y cualitativo buscan la homogeneización terminológica y del procedimiento descriptivo (Bruciamachie 1989, Bebi et al. 2001). La clave dicotómica define de forma objetiva tipos de masa discriminantes, interesantes, tanto para la gestión como para labores de investigación o seguimiento de rodales y su dinámica (Aubury et al. 1990).

La clave tipológica se complementa con una ficha descriptiva de grupo en la que se presentan las variables dasométricas descriptivas medias de cada tipo estructural, con lo que el inventario cualitativo puede sustituir a muy bajo coste al inventario cuantitativo tradicional en aquellas situaciones en las que la estimación de existencias con precisión no sea una variable trascendente como, por ejemplo, trabajos de diagnóstico silvícola, de estratificación o planes comarcales de ordenación de montes.

Las variables dasométricas descriptivas de los bosques bajos de Lomatio-Nothofagetum indican que -además del actual manejo silvopastoril- la implementación de modelos de gestión sostenible fundamentados en la obtención de biomasa leñosa puede ser una alternativa de manejo compatible con la presencia de ganadería extensiva.

Dentro de las líneas de investigación a abordar para definir la silvicultura de los ñirantales cabe citar como imperioso el estudio de la regeneración sexual de $N$. antarctica, reto imprescindible para garantizar la sostenibilidad y conservación de los ñirantales. Otros aspectos poco conocidos y de necesario análisis se encuentran en la respuesta de los ñirantales ante distintos escenarios silvícolas (intensidades de corta, rotaciones, respuestas a raleo, clasificación de productos, evaluación comparativa de producciones forrajeras, etc.), su valoración económica y viabilidad.

\section{AGRADECIMIENTOS}

Este trabajo fue financiado a través de los proyectos Regionales 61802-INTA, PICT08-14692 (SECyT) y del Plan de Movilidad del Personal Investigador de la Universidad de Valladolid.

\section{REFERENCIAS}

Amigo J, C Ramírez. 1998. A bioclimatic classification of Chile: woodland communities in the temperate zone. Plant Ecology 136: 9-26.

Assmann E. 1961. Waldertragskunde. München, Deutschland, BLV. 490 p.

Aubury S, M Bruciamacchie, P Druelle. 1990. L'inventaire typologique: Un outil performant pour l'elaboration des amenagements ou plans simples de gestion. Revue Forestière Francaise XLII (4): 429-444.

Bebi P, F Kienast, W Schönenberger. 2001. Assessing structures in mountain forests as a basis for investigating the forests dynamics and protective function. Forest Ecology and Management 145: 3-104.

Bruciamachie M. 1989. Typologie des peuplements. Revue Forestière Francaise XLI-6: 507- 512.

Cabrera A. 1976. Regiones Fitogeográficas Argentinas. In Enciclopedia Argentina de Agricultura y Jardinería. (Tomo II. $2^{\mathrm{a}}$ ed.). Buenos Aires, Argentina, ACME. 86 p.

Chauchard L, A González Peñalaba, L Maresca, A Rabino, R Sbrancia. 1995a. Dinámica y manejo del bosque de Nothofagus: (1) Crecimiento y mortalidad a nivel rodal. In Actas Jornadas Forestales Patagónicas 4, San Martín de los Andes, Argentina. p. 116-126.

Chauchard L, R Sbrancia, A González Peñalaba, A Rabino, L Maresca. 1995b. Dinámica y manejo del bosque de Nothofagus: (1) Aplicación de leyes fundamentales de la densidad de rodales. In Actas Jornadas Forestales Patagónicas 4, San Martín de los Andes, Argentina. p. 106-115.

Ciancio O, A Cuttini, R Mercurio, A Verancini. 1990. Un modèle sylvicole pour la conservation et l'amélioration de la pinède de Pin pignon d'Albarese (Toscana-Italy). Forêt méditerranéenne (XII 2): 131-142.

Claps L, A Schorr. 2005. Estudios económicos del manejo forestal en Patagonia. In SAyD. Alternativas de manejo sustentable para el manejo forestal integral de los bosques de Patagonia. Informe 2004-2005 del Proyecto Bosques Nativos y Áreas Protegidas (Proyecto PNUD ARG/ 99/011, Convenio de Préstamo No 4085 -AR BIRF). Buenos Aires, Argentina. Secretaría de Ambiente y Desarrollo Sustentable de la Nación.

Clutter J.L., J Fortson, L Penaar, G Brister, R Bailey. 1983. Timber management: A quantitative approach. New York, USA., John Willey \& Sons. 333 p.

Collado L. 2001. Los bosques de Tierra del Fuego. Análisis de su estratificación mediante imágenes satelitales para el inventario forestal de la provincia. Multequina 10: 01-16.

Donoso C. 1994. Bosques templados de Chile y Argentina. Variación, estructura y dinámica. Santiago de Chile, Chile. Universitaria. $484 \mathrm{p}$.

Dubourdieu J. 1991. L'intérêt de la conversion de taillis-sousfutaie es ses limites. Revue Forestière Francaise XLIII: 147-162.

Dubourdieu J. 1997. Manuel d'amenagement forestier. Paris, France. Office National de Forêts. 244 p.

Ducrey M. 1992. Quelle sylviculture et quel avenir pour les taillis de chêne vert (Quercus ilex L.) de la région méditerraneénne française. Revue Forestiére Française XLIV (1): $12-33$

Eskuche U. 1968. Fisionomía y sociología de los bosques de Nothofagus dombeyi en la región de Nahuel Huapi. Plant Ecology 16 (1-4): 192-204.

Eskuche U. 1969. Berberitzengebüsche und Nothofagus antarctica-wälder in Nordwestpatagonien. Plant Ecology 19 (1-6): 264-285.

Eskuche U. 1999. Estudios fitosociológicos en el norte de la Patagonia. II. Los bosques del Nothafagion dombeyi. Phytocoenologia 29(2): 177-252.

Fernández M, G Martínez-Pastur, F Boyeras, M Alloggia, R Vukasovic. 1995. Estimación de la producción de ñire en Tierra de Fuego: 1. Análisis de forma y ecuaciones locales de volumen para diferentes condiciones de sitio. In 
Actas Jornadas Forestales Patagónicas 4, San Martín de los Andes, Argentina. p. 137-144.

Gajardo R, F Luebert, M Estay. 2005. Ensayo de una tipología de estaciones forestales en el Parque Nacional Tolhuaca, Chile. Bosque 26 (2): 47-56.

Gajardo R. 1994. La vegetación natural de Chile. Clasificación y distribución geográfica. Santiago de Chile, Chile. Editorial Universitaria. $165 \mathrm{p}$.

Gallo L, P Marchell, P Crago, L Oubkrek, F Izquierdo, A Breitembücher, M González, L Chauchard, L Maresca, U Mele. 2000. Distribución y variación genética en características seminales y adaptativas de poblaciones y progenies de raulí en Argentina. In Ipinza R, B Gutiérrez, V Emhart (eds.). Domesticación y mejora genética de raulí y roble. Valdivia, Chile. Exion. p. 133-156.

Gómez I, G Gallopin. 1982. Estudio ecológico integrado de la cuenca del Río Manso Superior (Río Negro, Argentina). Las formas biológicas de las especies vasculares. Ecología Argentina 7: 117-126.

Hansen N, L Tejera, M Fertig. 2005. Sistemas silvopastorales en Chubut. In SAyD. Alternativas de manejo sustentable para el manejo forestal integral de los bosques de Patagonia. Informe 2004-2005 del Proyecto Bosques Nativos y Áreas Protegidas (Proyecto PNUD ARG/99/011, Convenio de Préstamo No 4085 -AR BIRF). Buenos Aires, Argentina. Secretaría de Ambiente y Desarrollo Sustentable de la Nación.

Hubert M. 1983. L'ammelioration des taillis par Balivage Intensif. Paris, France. Institute pour le Development Forestier. $117 \mathrm{p}$.

Ipinza R, B Gutiérrez, V Emhart (eds.). 2000. Domesticación y mejora genética de raulí y roble. Valdivia, Chile. Exion. 468 p.

Laclau P. 1997. Los ecosistemas forestales y el hombre en el sur de Chile y Argentina. Boletín Técnico Fundación Vida Silvestre (34).

Lanier L. 1986. Precis de sylviculture. Nancy, France. ENGREF. 486 p.

Lantschner V. 2005. Estructura de las comunidades de aves de los fondos de valles y laderas bajas de la cuenca del Río Foyel (Provincia de Río Negro), en relación a los disturbios antrópicos. Tesis de Licenciatura. Bariloche, Argentina. Centro Regional Universitario Bariloche, Universidad Nacional del Comahue, 116 p.

Lencinas MV, G Martínez-Pastur, JM Cellini, R Vukasovic, $\mathrm{P}$ Peri, M Fernández. 2002. Incorporación de la altura dominante y clase de sitio a ecuaciones estándar de volumen para Nothofagus antarctica (Forster f.) Oersted. Bosque 23 (2): 5-17.

Manacorda M, G Bonvisutto. 2001. Uso silvopastoral de los bosques de ñire (Nothofagus antarctica) en Río Negro, Patagonia Argentina. Revista Forestal Centroamericana 35: 41-44.

Martínez-Pastur G, M Fernández, F Boyeras, M Alloggia. 1995. Estimación de la producción para ñire en Tierra de Fuego: 2. Ecuaciones estándar de volumen. In Actas Jornadas Forestales Patagónicas 4, San Martín de los Andes, Argentina. p. 145-150.

Martínez-Pastur G, M Fernández. 1995. Estimación de la producción de ñire en Tierra de Fuego: 3. Ecuaciones de crecimiento para diferentes sitios y primeros resultados en la construcción de Índices de Sitio. In Actas Jornadas Forestales Patagónicas 4, San Martín de los Andes, Argentina. p. 1-11.

Montaña C. 1982. Las comunidades de ñire (Nothofagus antarctica (Forst.) Oerst.) en la cuenca del Río Manso Superior (Río Negro, Argentina). Tesis Doctoral. Córdoba, Argentina, Universidad Nacional de Córdoba, 134 p.

Oberdorfer E. 1960. Planzensoziologische Studien in Chile. Flora et Vegetatio II (III): 65-132.

Pardé L. 1930. Traité practique d'aménagement des forêts. Nancy - Paris, Presses universitaires de France. 547 p.

Pauchard A, E Ugarte, M Finckh, B Alaback. 2006. Subalpine Nothofagus and Araucaria forests across an environmental and disturbance gradient in South Central Chile: a floristic approach. [online]. Consultado jun. 2006. Disponible en http://www2.udec.cl/ pauchard/ conguillio.pdf.

Peri P, M Sturzenbaum, L Monelos, E Livraghi, R Christiansen, A Moreto, J Mayo. 2005 Productividad de sistemas silvopastoriles en bosques nativos de ñire (Nothofagus antarctica) de Patagonia Austral. In Actas III Congreso Forestal Argentino y Latinoamericano, Comisión Nuevas Tendencias Forestales. 10 p. (Presentación 11). Corrientes, 6-9 Septiembre 2005. (CD) ISSN 1669-6786.

Peri P. 2005. Sistemas silvopastoriles en ñirantales. IDIA XXI Revista de información sobre investigación y desarrollo agropecuario INTA.: 255-261.

Puntieri J, J Grosfeld, M Stecconi, C Brion, M Azpilicueta, L Gallo. 2006 Desarrollo temprano del roble (Nothofagus obliqua): un análisis arquitectural de procedencias de Argentina. Bosque 27(1): 44-51.

Ramírez C, M Correa, H Figueroa, J San Martín. 1985. Variación de hábito y hábitat de Nothofagus antarctica en el centro-sur de Chile. Bosque 6(2): 55-73.

Rusch V. 2005. Productividad y efectos ambientales en ñirantales: plantaciones con Pino oregón y sistemas silvopastoriles. Informe Proyecto Regional 61802-INTA (memoria año 2), Módulo 5: Biodiversidad, Bariloche, Argentina. INTA EEA-Bariloche. 5 p.

SAyD. 2005. Alternativas de manejo sustentable para el manejo forestal integral de los bosques de Patagonia. Informe 2004-2005 del Proyecto Bosques Nativos y Áreas Protegidas (Proyecto PNUD ARG/99/011, Convenio de Préstamo No 4085 -AR BIRF). Buenos Aires, Argentina. Secretaría de Ambiente y Desarrollo Sustentable de la Nación.

Schütz J. 1990. Sylviculture I. Lausanne, Suisse. Presses Polytechniques et Universitaires Romandes. 243 p.

SECF. 2005 (ed.). Diccionario Forestal. Madrid, España. Sociedad Española de Ciencias Forestales, Madrid, España. Ed. Mundi Prensa. 1314 p.

SilvaVoc 2006. SilvaTerm - Base de datos terminológica. I.U.F.R.O. (Internacional Union of Forest Research Organizations) [online]. Consultado dic. 2006. Disponible en http://www.iufro.org/

Somlo R, G Bonvisutto. 1995. Manejo silvopastoral en los bosques de ñire (Nothofagus antarctica) de la región de El Bolsón-Río Negro. Efectos de las diversas formas de intervención sobre la vegetación. In Actas Jornadas Forestales Patagónicas 4, San Martín de los Andes, Argentina. p. $42-55$. 
Somlo R, G Bonvisutto, T Schlichter, P Laclau, P Peri, M Allogia. 1997. Silvopastoral use of Argentine Patagonian Forests. In Gordon A, S Newman (eds.). Temperate agroforestry systems. CAB Internacional (UK). p. 237-250.

Veblen T, C Donoso, T Kitzberger, A Rebertus. 1996. Ecology of southern Chilean and Argentinean Nothofagus Forests. In Veblen, Hill, Read (eds). The ecology and biogeography of Nothofagus forests. New Haven, USA. Yale University Press. p. 293-353.
Veblen T, T Kitzberger, E Raffaele, D Lorenz. 2003. Fire history and vegetation changes in Northern Patagonia, Argentina. In Veblen T, W Baker, G Montenegro, T Swetnam (eds.). Fire and climatic changes in temperate ecosystems of the western Americas. New York, USA. Springer-Verlag. p. 265-293.

Willis B. 1914. El norte de la Patagonia. Comisión de Estudios Hidrológicos. Buenos Aires, Argentina, Ministerio de Obras Públicas, EUDEBA. 500 p.

Recibido: 29.08.06

Aceptado: 19.01.07 